\title{
Quantum Dots as Sources and Detectors of Mid- and Far-Infrared Radiation: Theoretical Models
}

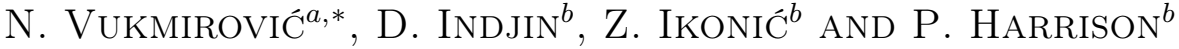 \\ ${ }^{a}$ Computational Research Division, Lawrence Berkeley National Laboratory, Berkeley, USA \\ ${ }^{b}$ School of Electronic and Electrical Engineering, University of Leeds, Leeds, UK
}

We present a review of theoretical methods used to study the electronic structure, optical and transport properties of intraband optoelectronic devices based on self-assembled quantum dots.

PACS numbers: 73.21.La, 78.67.Hc, 73.63.Kv, 85.60.Gz, 85.35.Be

\section{Introduction}

Most of the semiconductor optoelectronic devices utilize transitions between the state in the conduction band and the state in the valence band, so called interband transitions. The operating wavelength of such devices is mainly determined by the band gap of the material employed and is therefore limited to the near-infrared and visible part of the electromagnetic spectrum. However, if one wishes to access longer wavelengths, a different approach is required: the transitions within the same band (intraband transitions) have to be used. Since first-order optical transitions conserve the electron $\boldsymbol{k}$-vector, intraband optical transitions in bulk are not allowed and nanostructures have to be used instead. Therefore, in the last two decades, semiconductor nanostructures, such as quantum wells, wires and dots [1] have been recognized as sources and detectors of electromagnetic radiation in the mid- and far-infrared part of the spectrum. Those spectral regions are interesting due to a range of applications. The two atmospheric windows at $3-5 \mu \mathrm{m}$ and 8-13 $\mu \mathrm{m}$ where the atmosphere is transparent for electromagnetic radiation offer the possibility of free space optical communication, remote sensing and detection. Many molecular compounds have their vibrational modes in the 3-17 $\mu \mathrm{m}$ part of the spectrum and their detection enables the applications as measuring pollution, industrial process monitoring and detection of hidden explosives [2]. As many objects (including the human body) are the most emissive in this spectral region, corresponding detectors can be used for night vision. The far-infrared (terahertz) part of the spectrum can be potentially applied for medical imaging, astronomy and food quality control [3].

Following the advances in the growth of semiconductor quantum wells and superlattices, first intraband optoelectronic devices started to emerge in the 1980s and 1990s. The quantum well infrared photodetector (QWIP) [4] consists of a periodic array of quantum wells

* corresponding author; e-mail: NVukmirovic@lbl.gov subjected to an electric field perpendicular to the plane of the wells. Carriers from the ground state are excited to a higher state by absorbing incident photons. If the higher state is in the continuum or close to it, the excited carrier can be included in the transport and contributes to photocurrent. QWIPs can often be realized even with an array of single quantum wells. On the other hand, the quantum cascade laser (QCL) [5] consists of periodically replicated multiple quantum wells, that have to be very carefully engineered to direct the carrier transport through the desired states in such a way to enable optical gain in the structure. Realized QCL structures cover an impressively large spectral region from $\approx 3 \mu \mathrm{m}$ to $\approx 200 \mu \mathrm{m}$. Optically pumped intraband lasers [6], where the nonequilibrium distribution of carriers that enables the optical gain is achieved through optical pumping, have been realized as well. These are of limited practical interest since an external pump is required but can be very useful to understand the dynamics of carriers in the structure.

While achievements in the realization of a variety of quantum well intersubband devices are certainly impressive, the realized devices still have their limitations and one is facing the evergoing quest for the devices based on new concepts that would cover the so far unreachable parts of the spectrum and/or have an improved performance. For a number of reasons, that will be mentioned below, one expects that the use of self assembled quantum dots, instead of quantum wells, in the active region of intraband optoelectronic devices, can lead to significant improvements. Self assembled quantum dots are experimentally obtained during the growth of the layer of one material on top of another material with a different lattice constant if the width of the layer exceeds a certain critical thickness.

Several limitations of QWIPs have motivated the development of quantum dot infrared photodetectors (QDIPs) [7, 8]. The main origin of the undesirable dark current in QWIPs is thermal excitation (due to interaction with phonons) of the carriers from the ground state. The discrete electronic spectrum of quantum dots as op- 
posed to continuum spectrum of quantum wells significantly reduces the phase space for such processes and therefore reduces the dark current. Higher operating temperatures of QDIPs are therefore expected. Due to optical selection rules, QWIPs based on intersubband transitions in the conduction band interact only with radiation having the polarization vector in the growth direction ( $z$-direction, perpendicular to the plane of the quantum well). This is not the case in quantum dots since these are three-dimensional objects where such selection rules do not exist. The expected superior performance of QDIPs has recently indeed been demonstrated. QDIPs operating at room temperature have been demonstrated $[9,10]$, which is an exciting result since room temperature operation of QWIPs has not been achieved.

On the other hand, there has been less success in the development of quantum dot intraband lasers. In quantum well intraband lasers the lasing threshold depends on the lifetime of the upper laser level which is determined by scattering of electrons with phonons, where the most dominant ones are the longitudinal optical (LO) phonons, which are nearly dispersionless. In order to have a lower threshold this scattering has to be reduced. In quantum dots that have discrete energy levels this scattering is strongly reduced unless two energy levels are separated exactly by an LO phonon energy. Therefore, one expects that quantum dot based intraband lasers should have a much lower threshold [11]. There have been several attempts to realize quantum dot intraband lasers, which lead to the observation of electroluminescence [12-14], but not yet lasing. The lack of precise controllability of quantum dot geometry is probably the main reason why lasing has not yet been achieved. Experimental evidence that the system with truly discrete states should have a lower threshold current comes from extremely low threshold currents observed in quantum well based QCLs in strong magnetic fields [15], that act by discretizing the otherwise continuous quantum well spectrum [16]. It is however from the applied point of view necessary to avoid the use of high magnetic fields and have a system with truly discrete states.

Previous discussion indicates that the prospect of having quantum dot intraband devices superior to quantum well based ones, has not yet been fully exploited. Further development in this field certainly requires the technological improvements in the growth of quantum dot structures, but also requires theoretical understanding of physical processes in these devices that would guide their design. We will focus in what follows on the current status in theoretical understanding of the electronic, optical and transport properties of quantum dot intraband devices.

\section{Theoretical models of electrical and optical processes in quantum dots}

In order to understand the processes that take place in quantum dot intraband devices, the first necessary ingredient is to know the electronic energy level structure of quantum dots. Next, one needs to know how electrons interact with external electromagnetic radiation. The optical absorption cross-section describes the photon absorption probability in QDIPs, while the same quantity is of interest for lasers, as well, since it determines the optical gain. For both types of devices it is also of high interest to understand the transport of carriers through the device as they move from one energy level to another.

\subsection{Electronic structure}

A variety of theoretical approaches of different levels of complexity have been developed to treat the electronic structure of quantum dots. Self-assembled quantum dots typically have the diameter of the order of $15-30 \mathrm{~nm}$ and the height of the order 3-7 nm, therefore they can often consist even of millions of atoms. Such a large number of atoms makes the approaches based on density functional theory computationally unfeasible. Nevertheless, approaches that treat each atom in a quantum dot explicitly, such as the empirical pseudopotential method [17] and the tight-binding method [18] have been applied to quantum dots. Both of these methods are computationally demanding but are still feasible on present day computers.

For most of the electronic and optical properties of semiconductors and nanostructures based upon them, it is only a certain region of momentum space where carriers reside. The idea of the $\boldsymbol{k} \cdot \boldsymbol{p}$ method [19-22] and its simplest version, the effective mass method is to exploit this fact and make an expansion of the wave function in a limited number of bulk Bloch functions at some characteristic point in momentum space (usually the $\Gamma$ point) multiplied by the slowly varying envelope function. One then obtains the eigenvalue problem of the Hamiltonian, where the eigenvectors consist of $N$ envelope functions, where $N$ is the number of bands in the expansion. While possibly limited in the description of some subtle effects, the $\boldsymbol{k} \cdot \boldsymbol{p}$ method can inherently incorporate the effects of band mixing, strain, piezoelectricity, as well as the influence of external fields, keeping a lower computational cost (which can be further reduced by exploiting the symmetry of the Hamiltonian [23-26]) compared to atomistic methods. This is the reason why $\boldsymbol{k} \cdot \boldsymbol{p}$ is mostly used when modeling of optoelectronic devices is concerned.

\subsection{Intraband optical properties}

Once the electronic structure of the quantum dot has been obtained, one can use the wave functions and energies of the states to calculate the response of the quantum dot system to external electromagnetic radiation. For typical QDIP operating conditions, one can assume that electromagnetic radiation is only a weak excitation and treat it within first order perturbation theory, as well as consider only linear terms in radiation field. Furthermore, the wavelength of mid-infrared radiation is much larger than the size of a quantum dot, so one can assume that in a certain moment in time, the dot effectively sees 
a spatially constant electromagnetic field (the dipole approximation). Within these approximations, the optical absorption cross-section on a certain transition is simply proportional to the square of the momentum matrix element between the two states (see for example [25] for a detailed mathematical description). Since in typical QDIPs only the ground state is populated, the absorption spectrum from the ground state is of most interest.
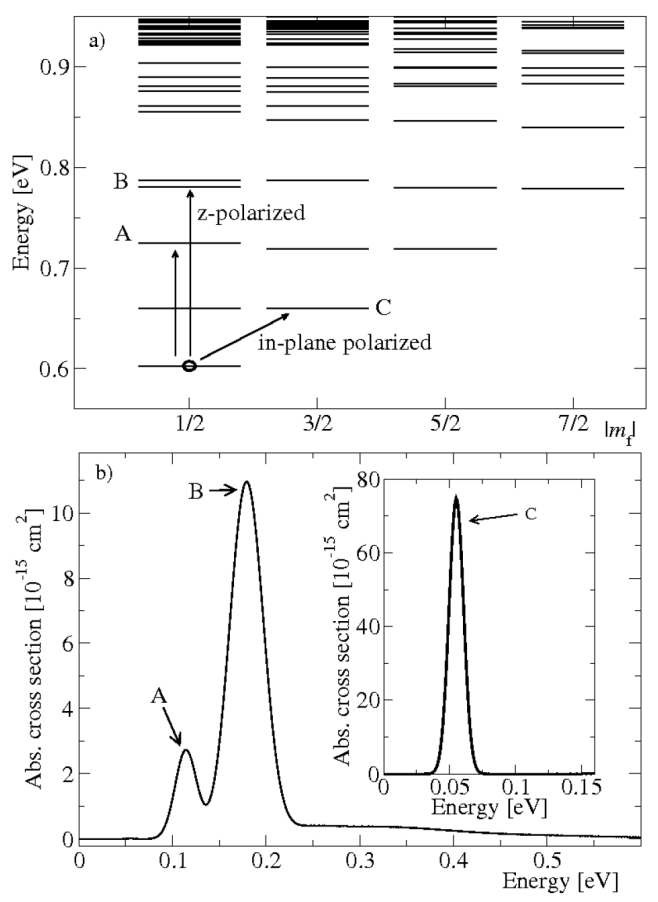

Fig. 1. (a) The scheme of energy levels and most relevant optical transitions of a cone shaped InAs/GaAs quantum dot with a diameter of $25 \mathrm{~nm}$ and the height of $7 \mathrm{~nm}$. (b) The intraband optical absorption cross-section from the ground state in the case of $z$-polarized radiation (main figure) and in-plane polarized radiation (inset).

In Fig. 1 we show the energy levels and optical absorption from the ground state for one typical quantum dot, calculated using the 8 band strain dependent $\boldsymbol{k} \cdot \boldsymbol{p}$ method. The results presented and other similar calculation suggest that the in-plane polarized radiation causes non-negligible transitions only between the ground and first excited state, these being located in the $\approx 40-80 \mathrm{meV}$ region in the far infrared. On the other hand, $z$-polarized radiation causes the transitions in the $\approx 100-300 \mathrm{meV}$ region in the mid-infrared. The origin of such behavior is actually the fact that the diameter of typical self-assembled quantum dots is much larger than their height. Such behavior can be altered only if the dot dimension in the $z$-direction becomes comparable to the in-plane dimensions [27]. In view of the fact that one of the expected advantages of QDIPs over QWIPs is the ability to detect radiation of any polarization, these results are somewhat discouraging. The ab- sorption of $z$-polarized radiation causing transition taking place to some higher excited state (possibly even in the continuum) can certainly be observed in photocurrent spectrum. However, in-plane polarized radiation causing transition to well bound first excited state(s) can hardly cause a strong photocurrent response. On the other hand, the mid-infrared response has been observed on many occasions in experiments performed in normal-incidence geometry $[7,28,29]$. This would contradict the results previously mentioned if one assumed ideally in-plane polarized radiation in these experiments. However, several effects (such as off-normal axis experimental missalignment, light scattering, etc.) can cause a certain degree of $z$-polarization in the radiation interacting with the dots, and cause the response in the mid-infrared $(\approx 100-300 \mathrm{meV})$.

\subsection{Transport properties}

Carrier transport through quantum nanostructures can be treated with different theoretical methodologies of different levels of complexity. Within the semiclassical Boltzmann equation approach, the carriers are treated as classical particles that jump from one energy level to another with a certain probability in a unit of time. This transition probability is on the other hand evaluated from quantum mechanical perturbation theory taking into account the interaction of carriers with phonons, impurities, etc. From the transition rates obtained in such a manner, one can form the equations that balance the inflow and outflow of carriers from a given state and solve them to obtain the populations of the states and the current through the structure [30]. In Fig. 2, we show the simulated responsivity (photocurrent divided by incident power) of a QDIP structure containing layers of quantum dots of the same dimensions as in Fig. 1, separated by $50 \mathrm{~nm}$ GaAs barriers. The comparison of Figs. $1 \mathrm{~b}$ and 2 illustrates the difference between the absorption and photocurrent spectrum. At a lower electric field, the peak, labeled as $\mathrm{A}$ in the absorption spectrum, is not present in the photocurrent spectrum. This is because the carriers are excited to a state which is too low in energy for carriers to reach the continuum states and form the photocurrent.

The most common approaches that treat the carriers on a fully quantum mechanical level to evaluate the transport are the density matrix approach [31, 32] and the nonequilibrium Green function approach [33, 34]. Within such approaches in addition to the occupation of states, one also considers the coherences between the states and therefore the number of variables that describe the system is much larger than in a semiclassical approach when one considers just the occupations. This makes these approaches computationally quite demanding especially when a large number of electronic states is involved. The nonequilibrium Green function method has been applied recently to study the feasibility of a quantum dot terahertz quantum cascade laser [35]. The results that predict low threshold current and high optical gain appear quite promising. 


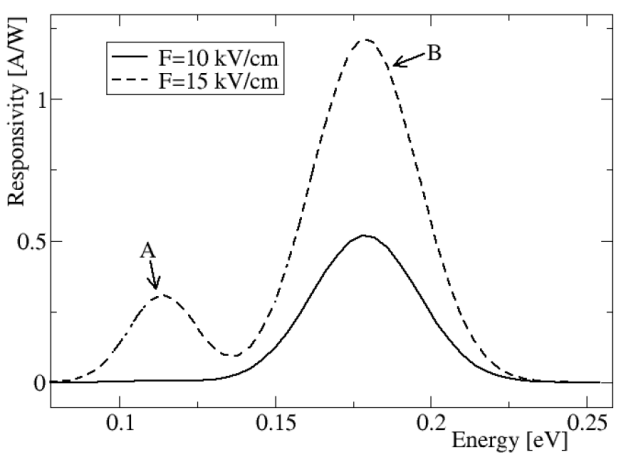

Fig. 2. The simulated dependence of the responsivity of the QDIP structure on the energy of incident photons. The temperature is $77 \mathrm{~K}$, and two different values of the electric field $F$ are considered.

\section{Conclusion}

To conclude, we have given an overview of the current state of research when the use of self-assembled quantum dots as detectors and lasers of mid- and far-infrared radiation is concerned. The theoretical approaches that treat the active region of these devices have been presented. One should note that for a full description of the devices one should also consider the carrier injection/extraction in the external contacts, as well as the appropriate waveguide in lasers and coupling of external radiation with active region in the detectors. These issues, being mostly rather general issues of optoelectronic devices, than specific issues related to quantum dot intraband devices, have not been covered here.

\section{References}

[1] P. Harrison, Quantum Wells, Wires and Dots, 2nd ed., Wiley, Chichester, England 2005.

[2] C. Gmachl, F. Capasso, D.L. Sivco, A.Y. Cho, Rep. Prog. Phys. 64, 1533 (2001).

[3] E. Pickwell, V.P. Wallace, J. Phys. D: Appl. Phys. 39, 301 (2006).

[4] B.F. Levine, K.K. Choi, C.G. Bethea, J. Walker, R.J. Malik, Appl. Phys. Lett. 50, 1092 (1987).

[5] J. Faist, F. Capasso, D.L. Sivco, C. Sirtori, A.L. Hutchinson, A.Y. Cho, Science 264, 553 (1994).

[6] O. Gauthier-Lafaye, P. Boucaud, F.H. Julien, S. Sauvage, S. Cabaret, J.-M. Lourtioz, V. Thierry-Mieg, R. Planel, Appl. Phys. Lett. 71, 3619 (1997).

[7] D. Pan, E. Towe, S. Kennerly, Appl. Phys. Lett. 73, 1937 (1998)

[8] S. Maimon, E. Finkman, G. Bahir, S.E. Schacham, J.M. Garcia, P.M. Petroff, Appl. Phys. Lett. 73, 2003 (1998).

[9] P. Bhattacharya, X.H. Su, S. Chakrabarti, G. Ariyawansa, A.G.U. Perera, Appl. Phys. Lett. 86, 191106 (2005).

[10] H. Lim, S. Tsao, W. Zhang, M. Razeghi, Appl. Phys. Lett. 90, 131112 (2007).
[11] N.S. Wingren, C.A. Stafford, IEEE J. Quantum Electron. 33, 1170 (1997)

[12] S. Anders, L. Rebohle, F.F. Schrey, W. Schrenk, K. Unterrainer, G. Strasser, Appl. Phys. Lett. $\mathbf{8 2}$, 3862 (2003)

[13] N. Ulbrich, J. Bauer, G. Scarpa, R. Boy, D. Schuh G. Abstreiter, S. Schmult, W. Wegscheider, Appl. Phys. Lett. 83, 1530 (2003).

[14] C.H. Fischer, P. Bhattacharya, P.C. Yu, Electron. Lett. 39, 1537 (2003).

[15] G. Scalari, S. Blaser, J. Faist, H. Beere, E. Linfield, D. Ritchie, G. Davies, Phys. Rev. Lett. 93, 237403 (2003).

[16] I. Savić, Z. Ikonić, V. Milanović, N. Vukmirović, V.D. Jovanović, D. Indjin, P. Harrison, Phys. Rev. B 73, 075321 (2006).

[17] J. Kim, L.-W. Wang, A. Zunger, Phys. Rev. B 57, R9408 (1998).

[18] T. Saito, J.N. Schulman, Y. Arakawa, Phys. Rev. B 57, 13016 (1998).

[19] O. Stier, M. Grundmann, D. Bimberg, Phys. Rev. B 59, 5688 (1999).

[20] C. Pryor, Phys. Rev. B 57, 7190 (1998).

[21] S. Tomić, A.G. Sunderland, I.J. Bush, J. Mater Chem. 16, 1963 (2006).

[22] V. Mlinar, M. Tadić, F.M. Peeters, Phys. Rev. B 73, 235336 (2006).

[23] N. Vukmirović, D. Indjin, V.D. Jovanović, Z. Ikonić, P. Harrison, Phys. Rev. B 72, 075356 (2005).

[24] N. Vukmirović, Z. Ikonić, D. Indjin, P. Harrison, J. Phys.: Condens. Matter 18, 6249 (2006).

[25] N. Vukmirović, Ž. Gačević, Z. Ikonić, D. Indjin, P. Harrison, V. Milanović, Semicond. Sci. Technol. 21, 1098 (2006).

[26] N. Vukmirović, D. Indjin, Z. Ikonić, P. Harrison, Appl. Phys. Lett. 88, 251107 (2006).

[27] H.C. Liu, Opto-Electron. Rev. 11, 1 (2003).

[28] L. Fu, H.H. Tan, I. McKerracher, J. Wong-Leung, C. Jagadish, N. Vukmirović, P. Harrison, J. Appl. Phys. 99, 114517 (2006).

[29] P. Aivaliotis, N. Vukmirović, E.A. Zibik, J.W. Cockburn, D. Indjin, P. Harrison, C. Groves, J.P.R. David, M. Hopkinson, L.R. Wilson, J. Phys. D: Appl. Phys. 40, 5537 (2007).

[30] N. Vukmirović, Z. Ikonić, I. Savić, D. Indjin, P. Harrison, J. Appl. Phys. 100, 074502 (2006).

[31] F. Rossi, T. Kuhn, Rev. Mod. Phys. 74, 895 (2002).

[32] I. Savić, N. Vukmirović, Z. Ikonić, D. Indjin, R.W. Kelsall, P. Harrison, V. Milanovic, Phys. Rev. B 76, 165310 (2007)

[33] A. Wacker, Phys. Rep. 357, 1 (2002).

[34] N. Vukmirović, Z. Ikonić, D. Indjin, P. Harrison, Phys. Rev. B 76, 245313 (2007).

[35] N. Vukmirović, D. Indjin, Z. Ikonić, P. Harrison, IEEE Phot. Technol. Lett. 20, 129 (2008). 